\title{
Almost sure central limit theorem for products of sums of partial sums
}

\author{
Fengxiang Feng ${ }^{1,2^{*}}$ and Dingcheng Wang ${ }^{1}$
}

\author{
"Correspondence: \\ fengfengxiang2013@163.com \\ 'school of Mathematical Science, \\ University of Electronic Science and \\ Technology of China, Chengdu, \\ Sichuan 611731, P.R. China \\ ${ }^{2}$ College of Science, Guilin \\ University of Technology, Guilin, \\ Guangxi 541004, P.R. China
}

\begin{abstract}
Considering a sequence of i.i.d. positive random variables, for products of sums of partial sums we establish an almost sure central limit theorem, which holds for some class of unbounded measurable functions.
\end{abstract}

MSC: $60 \mathrm{~F} 15$

Keywords: almost sure central limit theorem; products of sums of partial sums; unbounded measurable functions

\section{Introduction and main results}

Let $\left\{X_{n} ; n \geq 1\right\}$ be a sequence of random variables and define $S_{n}=\sum_{i=1}^{n} X_{i}$. Some results as regards the limit theorem of products $\prod_{j=1}^{n} S_{j}$ were obtained in recent years. Rempala and Wesolowski [1] obtained the following asymptotics for products of sums for a sequence of i.i.d. random variables.

Theorem A Let $\left\{X_{n} ; n \geq 1\right\}$ be a sequence of i.i.d. positive square integrable random variables with $\mathbb{E} X_{1}=\mu$, the coefficient of variation $\gamma=\sigma / \mu$, where $\sigma^{2}=\operatorname{Var}\left(X_{1}\right)$. Then

$$
\left(\frac{\prod_{k=1}^{n} S_{k}}{n ! \mu^{n}}\right)^{\frac{1}{\gamma \sqrt{n}}} \stackrel{d}{\rightarrow} e^{\sqrt{2} \mathcal{N}} \quad \text { as } n \rightarrow \infty
$$

Here and in the sequel, $\mathcal{N}$ is a standard normal random variable and $\stackrel{d}{\rightarrow}$ denotes the convergence in distribution.

Gonchigdanzan and Rempala [2] discussed the almost sure central limit theorem (ASCLT) for the products of partial sums and obtained the following result.

Theorem B Let $\left\{X_{n} ; n \geq 1\right\}$ be a sequence of i.i.d. positive random variables with $\mathbb{E} X_{1}=\mu$, $\operatorname{Var}\left(X_{1}\right)=\sigma^{2}$ the coefficient of variation $\gamma=\sigma / \mu$. Then

$$
\lim _{N \rightarrow \infty} \frac{1}{\log N} \sum_{n=1}^{N} \frac{1}{n} I\left\{\left(\frac{\prod_{k=1}^{n} S_{k}}{n ! \mu^{n}}\right)^{\frac{1}{\gamma \sqrt{n}}} \leq x\right\}=F(x) \quad \text { a.s. for any } x \in \mathbb{R}
$$

where $F$ is the distribution function of the random variable $e^{\sqrt{2} \mathcal{N}}$. Here and in the sequel, $I\{\cdot\}$ denotes the indicator function.

(c) 2016 Feng and Wang. This article is distributed under the terms of the Creative Commons Attribution 4.0 International License (http://creativecommons.org/licenses/by/4.0/), which permits unrestricted use, distribution, and reproduction in any medium, provided you give appropriate credit to the original author(s) and the source, provide a link to the Creative Commons license, and indicate if changes were made. 
Tan and Peng [3] proved the result of Theorem B still holds for some class of unbounded measurable functions and obtained the following result.

Theorem C Let $\left\{X_{n} ; n \geq 1\right\}$ be a sequence of i.i.d. positive random variables with $\mathbb{E} X_{1}=\mu$, $\operatorname{Var}\left(X_{1}\right)=\sigma^{2}, \mathbb{E}\left|X_{1}\right|^{3}<\infty$, the coefficient of variation $\gamma=\sigma / \mu$. Let $g(x)$ be a real valued almost everywhere continuous function on $\mathbb{R}$ such that $\left|g\left(e^{x}\right) \phi(x)\right| \leq c(1+|x|)^{-\alpha}$ with some $c>0$ and $\alpha>5$. Then

$$
\lim _{N \rightarrow \infty} \frac{1}{\log N} \sum_{n=1}^{N} \frac{1}{n} g\left\{\left(\frac{\prod_{k=1}^{n} S_{k}}{n ! \mu^{n}}\right)^{\frac{1}{\gamma \sqrt{n}}}\right\}=\int_{0}^{\infty} g(x) \mathrm{d} F(x) \quad \text { a.s. for any } x \in \mathbb{R},
$$

where $F(\cdot)$ is the distribution function of the random variable $e^{\sqrt{2} \mathcal{N}}$ and $\phi(x)$ is the density function of the standard normal random variable.

Zhang et al. [4] discussed the almost sure central limit theory for products of sums of partial sums and obtained the following result.

Theorem $\mathbf{D}$ Let $\left\{X, X_{n} ; n \geq 1\right\}$ be a sequence of i.i.d. positive square integrable random variables with $\mathbb{E} X=\mu, \operatorname{Var}(X)=\sigma^{2}<\infty$, the coefficient of variation $\gamma=\sigma / \mu$. Denote $S_{n}=$ $\sum_{i=1}^{n} X_{i}, T_{k}=\sum_{i=1}^{k} S_{i}$. Then

$$
\lim _{n \rightarrow \infty} \frac{1}{\log n} \sum_{k=1}^{n} \frac{1}{k} I\left\{\left(\frac{2^{k} \prod_{j=1}^{k} T_{j}}{k !(k+1) ! \mu^{k}}\right)^{\frac{1}{\gamma \sqrt{k}}} \leq x\right\}=F(x) \quad \text { a.s. for any } x \in \mathbb{R},
$$

where $F(\cdot)$ is the distribution function of the random variable $e^{\sqrt{10 / 3} \mathcal{N}}$.

The purpose of this article is to establish that Theorem D holds for some class of unbounded measurable functions.

Our main result is the following theorem.

Theorem 1.1 Let $\left\{X_{n} ; n \geq 1\right\}$ be a sequence of i.i.d. positive random variables with $\mathbb{E} X_{1}=\mu$, $\operatorname{Var}\left(X_{1}\right)=\sigma^{2}, \mathbb{E}\left|X_{1}\right|^{3}<\infty$, the coefficient of variation $\gamma=\sigma / \mu$. Let $g(x)$ be a real valued almost everywhere continuous function on $\mathbb{R}$ such that $\left|g\left(e^{\sqrt{10 / 3} x}\right) \phi(x)\right| \leq c(1+|x|)^{-\alpha}$ with some $c>0$ and $\alpha>5$. Denote $S_{n}=\sum_{i=1}^{n} X_{i}, T_{k}=\sum_{i=1}^{k} S_{i}$. Then

$$
\begin{gathered}
\lim _{N \rightarrow \infty} \frac{1}{\log N} \sum_{n=1}^{N} \frac{1}{n} g\left(\left(\frac{2^{n} \prod_{k=1}^{n} T_{k}}{n !(n+1) ! \mu^{n}}\right)^{\frac{1}{\gamma \sqrt{n}}}\right) \\
=\int_{0}^{\infty} g(x) \mathrm{d} F(x) \quad \text { a.s. for any } x \in \mathbb{R},
\end{gathered}
$$

where $F(\cdot)$ is the distribution function of the random variable $e^{\sqrt{10 / 3} \mathcal{N}}$. Here and in the sequel, $\phi(x)$ is the density function of the standard normal random variable.

Remark 1 Let $f(x)=g\left(e^{\sqrt{10 / 3} x}\right), t=e^{\sqrt{10 / 3} x}$. Then

$$
x=\sqrt{\frac{3}{10}} \log t, \quad g(t)=f\left(\sqrt{\frac{3}{10}} \log t\right),
$$




$$
\begin{aligned}
g\left(\left(\frac{2^{n} \prod_{k=1}^{n} T_{k}}{n !(n+1) ! \mu^{n}}\right)^{\frac{1}{\gamma \sqrt{n}}}\right) & =f\left(\sqrt{\frac{3}{10}} \log \left(\prod_{k=1}^{n} \frac{2 T_{k}}{k(k+1) \mu}\right)^{\frac{1}{\gamma \sqrt{n}}}\right) \\
& =f\left(\frac{1}{\gamma \sqrt{10 n / 3}} \sum_{k=1}^{n} \log \frac{T_{k}}{k(k+1) \mu / 2}\right)
\end{aligned}
$$

Since $F(x)$ is the distribution function of the random variable $e^{\sqrt{10 / 3} \mathcal{N}}$, we can get $F(x)=$ $\Phi\left(\sqrt{\frac{3}{10}} \log x\right)$, where $\Phi(x)$ is the distribution function of the standard normal random variable. Hence we have the following: Let $f(x)=g\left(e^{\sqrt{10 / 3} x}\right)$ and $f(x)$ be a real valued almost everywhere continuous function on $\mathbb{R}$ such that $|f(x) \phi(x)| \leq c(1+|x|)^{-\alpha}$ with some $c>0$ and $\alpha>5$, then (1.5) is equivalent to

$$
\begin{aligned}
& \lim _{N \rightarrow \infty} \frac{1}{\log N} \sum_{n=1}^{N} \frac{1}{n} f\left(\frac{1}{\gamma \sqrt{10 n / 3}} \sum_{k=1}^{n} \log \frac{T_{k}}{k(k+1) \mu / 2}\right) \\
& \quad \int_{-\infty}^{\infty} f(x) \phi(x) \mathrm{d} x \quad \text { a.s. for any } x \in \mathbb{R} .
\end{aligned}
$$

Remark 2 By the proof of Theorem 2 of Berkes et al. [5], in order to prove (1.5), it suffices to show (1.6) holds true for $f(x) \phi(x)=(1+|x|)^{-\alpha}$ with $\alpha>5$. Here and in the sequel, $f(x)$ satisfies $f(x) \phi(x)=(1+|x|)^{-\alpha}$ with $\alpha>5$.

\section{Preliminaries}

In the following, the notation $a_{n} \sim b_{n}$ means that $\lim _{n \rightarrow \infty} a_{n} / b_{n}=1$ and $a_{n} \ll b_{n}$ means that $\limsup _{n \rightarrow \infty}\left|a_{n}\right| b_{n} \mid<+\infty$. We denote $b_{k, n}=\sum_{j=k}^{n} \frac{1}{j}, c_{k, n}=2 \sum_{j=k}^{n} \frac{j+1-k}{j(j+1)}, d_{k, n}=\frac{n+1-k}{n+1}$, $\widetilde{X}_{i}=\frac{X_{i}-\mu}{\sigma}, \widetilde{S}_{k}=\sum_{i=1}^{k} \widetilde{X}_{i}, S_{k, n}=\sum_{i=1}^{k} c_{i, n} \widetilde{X}_{i}$. By Lemma 2.1 of Wu [6], we can get

$$
c_{i, n}=2\left(b_{i, n}-d_{i, n}\right), \quad \sum_{i=1}^{n} c_{i, n}^{2} \sim \frac{10 n}{3} .
$$

Let

$$
Y_{i}=\frac{1}{\gamma \sqrt{10 i / 3}} \sum_{k=1}^{i} \log \frac{T_{k}}{k(k+1) \mu / 2} .
$$

Note that

$$
\begin{aligned}
& \frac{1}{\gamma} \sum_{k=1}^{i}\left(\frac{T_{k}}{k(k+1) \mu / 2}-1\right) \\
& \quad=\frac{1}{\gamma} \sum_{k=1}^{i}\left(\frac{2 \sum_{j=1}^{k} S_{j}-k(k+1) \mu}{k(k+1) \mu}\right) \\
& =\frac{1}{\gamma} \sum_{k=1}^{i} \frac{2}{k(k+1) \mu} \sum_{j=1}^{k} \sum_{l=1}^{j}\left(X_{l}-\mu\right) \\
& \quad=\frac{1}{\gamma} \sum_{k=1}^{i} \frac{2}{k(k+1) \mu} \sum_{l=1}^{k} \sum_{j=l}^{k}\left(X_{l}-\mu\right)
\end{aligned}
$$




$$
\begin{aligned}
& =\frac{\mu}{\sigma} \sum_{k=1}^{i} \frac{2}{k(k+1) \mu} \sum_{l=1}^{k}(k+1-l)\left(X_{l}-\mu\right) \\
& =\sum_{k=1}^{i} \sum_{l=1}^{k} \frac{2(k+1-l)}{k(k+1)} \frac{X_{l}-\mu}{\sigma} \\
& =\sum_{l=1}^{i} \sum_{k=l}^{i} \frac{2(k+1-l)}{k(k+1)} \widetilde{X}_{l} \\
& =\sum_{l=1}^{i} c_{l, i} \widetilde{X}_{l}=S_{i, i} .
\end{aligned}
$$

By the fact that $\log (1+x)=x+\frac{\delta}{2} x^{2}$, where $|x|<1, \delta \in(-1,0)$, thus we have

$$
\begin{aligned}
Y_{i} & =\frac{1}{\gamma \sqrt{10 i / 3}} \sum_{k=1}^{i} \log \frac{T_{k}}{k(k+1) \mu / 2} \\
& =\frac{1}{\gamma \sqrt{10 i / 3}} \sum_{k=1}^{i}\left(\frac{T_{k}}{k(k+1) \mu / 2}-1\right)+\frac{1}{\gamma \sqrt{10 i / 3}} \sum_{k=1}^{i} \frac{\delta_{k}}{2}\left(\frac{T_{k}}{k(k+1) \mu / 2}-1\right)^{2} \\
& =\frac{1}{\sqrt{10 i / 3}} S_{i, i}+\frac{1}{\gamma \sqrt{10 i / 3}} \sum_{k=1}^{i} \frac{\delta_{k}}{2}\left(\frac{T_{k}}{k(k+1) \mu / 2}-1\right)^{2} \\
& =: \frac{1}{\sqrt{10 i / 3}} S_{i, i}+R_{i} .
\end{aligned}
$$

By the fact that $\mathbb{E}\left|X_{1}\right|^{2}<\infty$, using the Marcinkiewicz-Zygmund strong large number law, we have

$$
\begin{aligned}
& S_{k}-k \mu=o\left(k^{1 / 2}\right) \quad \text { a.s., } \\
&\left|\frac{T_{k}}{k(k+1) \mu / 2}-1\right|=\left|\frac{2 \sum_{j=1}^{k} S_{j}-k(k+1) \mu}{k(k+1) \mu}\right| \\
& \leq \frac{2\left|\sum_{j=1}^{k}\left(S_{j}-j \mu\right)\right|}{k(k+1) \mu} \\
& \leq \frac{2 \sum_{j=1}^{k} j^{1 / 2}}{k(k+1) \mu} \ll \frac{k^{3 / 2}}{k^{2}}=\frac{1}{k^{1 / 2}} .
\end{aligned}
$$

Thus

$$
\left|R_{i}\right| \ll \frac{1}{\sqrt{i}} \sum_{k=1}^{i} \frac{1}{k} \ll \frac{\log i}{\sqrt{i}} \quad \text { a.s. }
$$

In order to prove Theorem 1.1, we introduce the following lemmas.

Lemma 2.1 Let $X$ and $Y$ be random variables. Set $F(x)=P(X<x), G(x)=P(X+Y<x)$, then for any $\varepsilon>0$ and $x \in \mathbb{R}$,

$$
F(x-\varepsilon)-P(|Y| \geq \varepsilon) \leq G(x) \leq F(x+\varepsilon)+P(|Y| \geq \varepsilon) .
$$


Proof See Lemma 3 on p.16 of Petrov [7].

Lemma 2.2 Let $\left\{X_{n} ; n \geq 1\right\}$ be a sequence of i.i.d. positive random variables. Denote $S_{n}=$ $\sum_{i=1}^{n} X_{i}, F^{s}$ denotes the distribution function obtained from $F$ by symmetrization and choose $L>0$ so large that $\int_{|x| \leq L} x^{2} \mathrm{~d} F^{s}(x) \geq 1$. Then, for any $n \geq 1, \lambda>0$, there exists a $c>0$ such that

$$
\sup _{a} P\left(a \leq \frac{S_{n}}{\sqrt{n}} \leq a+\lambda\right) \leq c \lambda
$$

holds for $\lambda \sqrt{n} \geq L$.

Proof See (20) on p.73 of Berkes et al. [5].

Let

$$
\begin{aligned}
& Z_{k}=\sum_{i=2^{k}+1}^{2^{k+1}} \frac{1}{i} f\left(Y_{i}\right), \\
& Z_{k}^{*}=\sum_{i=2^{k}+1}^{2^{k+1}} \frac{1}{i} f\left(Y_{i}\right) I\left\{f\left(Y_{i}\right) \leq \frac{k}{(\log k)^{\beta}}\right\},
\end{aligned}
$$

where $1<\beta<(\alpha-3) / 2$.

Lemma 2.3 Under the conditions of Theorem 1.1, we get

$$
\mathbb{P}\left(Z_{k} \neq Z_{k}^{*}, \text { i.o. }\right)=0 \text {. }
$$

Proof It is easy to get

$$
\begin{aligned}
\left\{Z_{k} \neq Z_{k}^{*}\right\} \subseteq & \left\{\left|Y_{i}\right| \geq f^{-1}\left(k /(\log k)^{\beta}\right) \text { for some } 2^{k}<i \leq 2^{k+1}\right\} \\
= & \left\{\left|\frac{1}{\sqrt{10 i / 3}} S_{i, i}+R_{i}\right| \geq f^{-1}\left(k /(\log k)^{\beta}\right) \geq(2 \log k+(\alpha-2 \beta) \log \log k)^{1 / 2}\right. \\
& \text { for some } \left.2^{k}<i \leq 2^{k+1}\right\} .
\end{aligned}
$$

Since $\left|R_{i}\right| \ll \frac{\log i}{\sqrt{i}}$ a.s.; see (2.1). By the law of iterated logarithm (Feller [8], Theorem 2), we get

$$
\begin{aligned}
\mathbb{P}\left(Z_{k} \neq Z_{k}^{*}, \text { i.o. }\right) & \leq \mathbb{P}\left(\left|\frac{1}{\sqrt{10 i / 3}} S_{i, i}\right| \geq(2 \log \log i+(\alpha-2 \beta) \log \log \log i-O(1))^{1 / 2} \text {,i.o. }\right) \\
& =0
\end{aligned}
$$

We complete the proof of Lemma 2.3.

Let $G_{i}, F_{i}, F$ denote the distribution functions of $Y_{i}, \frac{\widetilde{S}_{i}}{\sqrt{i}}, \widetilde{X}_{1}$, respectively. $\Phi$ denotes the distribution function of the standard normal distribution function. Set

$$
\sigma_{i}^{2}=\int_{-\sqrt{i}}^{\sqrt{i}} x^{2} \mathrm{~d} F(x)-\left(\int_{-\sqrt{i}}^{\sqrt{i}} x \mathrm{~d} F(x)\right)^{2}
$$




$$
\varepsilon_{i}=\sup _{x}\left|F_{i}(x)-\Phi\left(\frac{x}{\sigma_{i}}\right)\right|, \quad \theta_{i}=\sup _{x}\left|G_{i}(x)-\Phi\left(\frac{x}{\sigma_{i}}\right)\right| .
$$

Obviously $\sigma_{i} \leq 1, \lim _{i \rightarrow \infty} \sigma_{i}=1$.

Lemma 2.4 Under the conditions of Theorem 1.1, we have

$$
\sum_{k=1}^{N} \mathbb{E}\left(Z_{k}^{*}\right)^{2} \ll \frac{N^{2}}{(\log N)^{2 \beta}}
$$

Proof Note that the estimation

$$
\left|\int_{-a}^{a} \Psi(x) \mathrm{d}\left(H_{1}(x)-H_{2}(x)\right)\right| \leq \sup _{-a \leq x \leq a}|\Psi(x)| \cdot \sup _{-a \leq x \leq a}\left|H_{1}(x)-H_{2}(x)\right|
$$

holds for any bounded, measurable function $\Psi(x)$ and the distribution functions $H_{1}(x)$, $H_{2}(x)$. Thus for $2^{k}<i \leq 2^{k+1}$, we get

$$
\begin{aligned}
& \mathbb{E} f^{2}\left(Y_{i}\right) I\left\{f\left(Y_{i}\right) \leq \frac{k}{(\log k)^{\beta}}\right\} \\
& \quad=\int_{|x| \leq a_{k}} f^{2}(x) \mathrm{d} G_{i}(x) \\
& \quad \leq \int_{|x| \leq a_{k}} f^{2}(x) \mathrm{d} \Phi\left(\frac{x}{\sigma_{i}}\right)+\theta_{i} \frac{k^{2}}{(\log k)^{2 \beta}} \\
& \quad \ll \int_{|x| \leq a_{k}} f^{2}(x) \mathrm{d} \Phi(x)+\theta_{i} \frac{k^{2}}{(\log k)^{2 \beta}} ;
\end{aligned}
$$

here and in the sequel $a_{k}=f^{-1}\left(\frac{k}{(\log k)^{\beta}}\right)$. Hence, by the Cauchy-Schwarz inequality and the fact that $f(x) \phi(x)=(1+|x|)^{-\alpha}$, we obtain

$$
\begin{aligned}
\mathbb{E}\left(Z_{k}^{*}\right)^{2} & \ll \mathbb{E}\left(\left(\sum_{i=2^{k}+1}^{2^{k+1}}\left(\frac{1}{i}\right)^{2}\right)^{1 / 2}\left(\sum_{i=2^{k}+1}^{2^{k+1}} f^{2}\left(Y_{i}\right) I\left\{f\left(Y_{i}\right) \leq \frac{k}{(\log k)^{\beta}}\right\}\right)^{1 / 2}\right)^{2} \\
& \ll\left(\sum_{i=2^{k}+1}^{2^{k+1}} \frac{1}{i^{2}}\right)\left(\sum_{i=2^{k}+1}^{2^{k+1}}\left(\int_{|x| \leq a_{k}} f^{2}(x) \mathrm{d} \Phi(x)+\theta_{i} \frac{k^{2}}{(\log k)^{2 \beta}}\right)\right) \\
& \ll \frac{1}{2^{k}}\left(2^{k} \int_{|x| \leq a_{k}} f^{2}(x) \mathrm{d} \Phi(x)+\frac{k^{2}}{(\log k)^{2 \beta}} \sum_{i=2^{k}+1}^{2^{k+1}} \theta_{i}\right) \\
& \ll \int_{|x| \leq a_{k}} \frac{e^{x^{2} / 2}}{(1+|x|)^{2 \alpha}} \mathrm{d} x+\frac{k^{2}}{(\log k)^{2 \beta}} \sum_{i=2^{k}+1}^{2^{k+1}} \frac{\theta_{i}}{i}
\end{aligned}
$$

By the same methods as that on p.72 of Berkes et al. [5], we get

$$
\int_{|x| \leq a_{k}} \frac{e^{x^{2} / 2}}{(1+|x|)^{2 \alpha}} \mathrm{d} x \ll \frac{k}{(\log k)^{\beta+(\alpha+1) / 2}} .
$$


Now we estimate $\theta_{i}$. By Lemma 2.1, for any $\varepsilon>0$, we have

$$
\begin{aligned}
\theta_{i}= & \sup _{x}\left|G_{i}(x)-\Phi\left(\frac{x}{\sigma_{i}}\right)\right| \\
\leq & \sup _{x}\left|G_{i}(x)-F_{i}(x)\right|+\sup _{x}\left|F_{i}(x)-\Phi\left(\frac{x}{\sigma_{i}}\right)\right| \\
= & \sup _{x}\left|P\left(Y_{i} \leq x\right)-P\left(\frac{\widetilde{S}_{i}}{\sqrt{i}} \leq x\right)\right|+\varepsilon_{i} \\
\leq & \sup _{x}\left|P\left(Y_{i} \leq x\right)-P\left(\frac{S_{i, i}}{\sqrt{10 i / 3}} \leq x\right)\right|+\sup _{x}\left|P\left(\frac{S_{i, i}}{\sqrt{10 i / 3}} \leq x\right)-P\left(\frac{\widetilde{S}_{i}}{\sqrt{i}} \leq x\right)\right|+\varepsilon_{i} \\
\leq & \sup _{x}\left|P\left(\frac{S_{i, i}}{\sqrt{10 i / 3}}+R_{i} \leq x\right)-P\left(\frac{S_{i, i}}{\sqrt{10 i / 3}} \leq x+\varepsilon\right)\right| \\
& +\sup _{x}\left|P\left(\frac{S_{i, i}}{\sqrt{10 i / 3}} \leq x+\varepsilon\right)-P\left(\frac{S_{i, i}}{\sqrt{10 i / 3}} \leq x\right)\right| \\
& +\sup _{x}\left|P\left(\frac{S_{i, i}}{\sqrt{10 i / 3}} \leq x\right)-P\left(\frac{\widetilde{S}_{i}}{\sqrt{i}} \leq x\right)\right|+\varepsilon_{i} \\
& \quad P\left(\left|R_{i}\right| \geq \varepsilon\right)+\sup _{x}\left|P\left(\frac{S_{i, i}}{\sqrt{10 i / 3}} \leq x+\varepsilon\right)-P\left(\frac{S_{i, i}}{\sqrt{10 i / 3}} \leq x\right)\right| \\
& +\sup _{x}\left|P\left(\frac{S_{i, i}}{\sqrt{10 i / 3}} \leq x\right)-P\left(\frac{\widetilde{S}_{i}}{\sqrt{i}} \leq x\right)\right|+\varepsilon_{i} .
\end{aligned}
$$

By the Markov inequality and (2.1), we have

$$
P\left(\left|R_{i}\right| \geq \varepsilon\right) \leq \frac{\mathbb{E}\left|R_{i}\right|}{\varepsilon} \ll \frac{\log i}{\sqrt{i \varepsilon}} .
$$

By Lemma 2.2, we have

$$
\sup _{x}\left|P\left(\frac{S_{i, i}}{\sqrt{10 i / 3}} \leq x+\varepsilon\right)-P\left(\frac{S_{i, i}}{\sqrt{10 i / 3}} \leq x\right)\right| \ll \varepsilon .
$$

By the Berry-Esseen inequality, we have

$$
\begin{aligned}
& \sup _{x}\left|P\left(\frac{S_{i, i}}{\sqrt{10 i / 3}} \leq x\right)-P\left(\frac{\widetilde{S}_{i}}{\sqrt{i}} \leq x\right)\right| \\
& \leq \sup _{x}\left|P\left(\frac{S_{i, i}}{\sqrt{10 i / 3}} \leq x\right)-\Phi(x)\right|+\sup _{x}\left|P\left(\frac{\widetilde{S}_{i}}{\sqrt{i}} \leq x\right)-\Phi(x)\right| \\
& \ll \frac{1}{i^{1 / 2}}+\frac{1}{i^{1 / 2}} .
\end{aligned}
$$

Let $\varepsilon=i^{-1 / 3}$, then

$$
\theta_{i} \ll \frac{\log i}{i^{1 / 6}}+\frac{1}{i^{1 / 3}}+\frac{1}{i^{1 / 2}}+\varepsilon_{i}
$$

Therefore, there exists $\varepsilon_{0}>0$ such that

$$
\theta_{i} \ll \frac{1}{i^{\varepsilon_{0}}}+\varepsilon_{i} .
$$


By Theorem 1 of Friedman et al. [9], we have

$$
\sum_{i=1}^{\infty} \frac{\varepsilon_{i}}{i}<\infty
$$

Hence

$$
\sum_{i=1}^{\infty} \frac{\theta_{i}}{i} \ll \sum_{i=1}^{\infty} \frac{\frac{1}{i^{1} 0}+\varepsilon_{i}}{i}<\infty .
$$

By the fact that $(\alpha+1) / 2>\beta$, we have

$$
\sum_{k=1}^{N} \mathbb{E}\left(Z_{k}^{*}\right)^{2} \ll \sum_{k=1}^{N} \frac{k}{(\log k)^{\beta+(\alpha+1) / 2}}+\sum_{k=1}^{N} \frac{k^{2}}{(\log k)^{2 \beta}} \sum_{i=2^{k}+1}^{2^{k+1}} \frac{\theta_{i}}{i} \ll \frac{N^{2}}{(\log N)^{2 \beta}} .
$$

We complete the proof of Lemma 2.4.

Lemma 2.5 Under the conditions of Theorem 1.1, for $l \geq l_{0}$, we have

$$
\left|\operatorname{Cov}\left(Z_{k}^{*}, Z_{l}^{*}\right)\right| \ll \frac{k l}{(\log k)^{\beta}(\log l)^{\beta}} 2^{-(l-k) \tau},
$$

where $\tau$ is a constant $0<\tau \leq 1 / 8$.

Proof For $1 \leq i \leq j / 2, j \geq j_{0}$ and any $x, y$, we first prove

$$
\left|P\left(Y_{i} \leq x, Y_{j} \leq y\right)-P\left(Y_{i} \leq x\right) P\left(Y_{j} \leq y\right)\right| \ll\left(\frac{i}{j}\right)^{\tau} .
$$

Let $\rho=\frac{i}{j}$. By the Chebyshev inequality, we have

$$
P\left(\left|\frac{S_{i, i}}{\sqrt{10 j / 3}}\right| \geq \rho^{1 / 8}\right)=P\left(\left|\frac{S_{i, i}}{\sqrt{10 i / 3}}\right| \geq \sqrt{\frac{j}{i}} \rho^{1 / 8}\right) \leq \frac{i}{j} \rho^{-1 / 4} \leq \rho^{1 / 8} \leq \rho^{\tau_{1}},
$$

where $\tau_{1}$ is a constant $0<\tau_{1} \leq 1 / 8$.

By the Markov inequality and (2.1), for $j \geq j_{0}$, we have

$$
P\left(\left|R_{j}\right| \geq \rho^{1 / 8}\right) \leq \frac{\mathbb{E}\left|R_{j}\right|}{\rho^{1 / 8}} \ll \frac{\log j}{j^{1 / 2} \rho^{1 / 8}}=\rho^{1 / 8} \frac{\log j}{j^{1 / 4} i^{1 / 4}} \ll \rho^{\tau_{2}},
$$

where $\tau_{2}$ is a constant, $0<\tau_{2} \leq 1 / 8$.

By the Markov inequality, we have

$$
\begin{aligned}
& P\left(\left|\sqrt{1-\rho} \frac{c_{i+1, j} \widetilde{S}_{i}}{\sqrt{10(j-i) / 3}}\right| \geq \rho^{1 / 8}\right) \\
& \quad=P\left(\left|\frac{\widetilde{S}_{i}}{\sqrt{i}}\right| \geq \sqrt{\frac{10 / 3 j}{i}} \frac{1}{c_{i+1, j}} \rho^{1 / 8}\right) \\
& \quad \leq \frac{3}{10} \rho^{3 / 4}\left(c_{i+1, j}\right)^{2}=\frac{3}{10} \rho^{3 / 4}\left(2 b_{i+1, j}-2 d_{i+1, j}\right)^{2}
\end{aligned}
$$




$$
\begin{aligned}
& =\frac{3}{10} \rho^{3 / 4}\left[\left(2 \sum_{k=i+1}^{j} \frac{1}{k}\right)^{2}+4\left(\frac{j+1-i-1}{j+1}\right)^{2}-8\left(\sum_{k=i+1}^{j} \frac{1}{k}\right) \frac{j+1-i-1}{j+1}\right] \\
& \ll \rho^{3 / 4}\left[\left(\log \frac{j}{i}\right)^{2}+\left(\frac{j-i}{j+1}\right)^{2}-\frac{j-i}{j+1} \log \frac{j}{i}\right] \\
& \ll \rho^{\tau_{3}},
\end{aligned}
$$

where $\tau_{3}$ is a constant that satisfies $0<\tau_{3} \leq 1 / 8$.

By Lemma 2.2 and the fact that $\rho=\frac{i}{j}, 1 \leq i \leq j / 2$, we have

$$
P\left(y-3 \rho^{1 / 8} \leq \sqrt{1-\rho} \frac{S_{j, j}-S_{i, i}-c_{i+1, j} \widetilde{S}_{i}}{\sqrt{10(j-i) / 3}} \leq y\right) \ll \frac{\rho^{1 / 8}}{\sqrt{1-\rho}} \ll \rho^{1 / 8} .
$$

Set $\tau=\min \left\{\tau_{1}, \tau_{2}, \tau_{3}, 1 / 8\right\}$, we get

$$
\begin{aligned}
P\left(Y_{i} \leq x, Y_{j} \leq y\right) & \\
= & P\left(Y_{i} \leq x, \frac{S_{j, j}}{\sqrt{10 j / 3}}+R_{j} \leq y\right) \\
= & P\left(Y_{i} \leq x, \frac{S_{i, i}}{\sqrt{10 j / 3}}+\sqrt{1-\rho} \frac{S_{j, j}-S_{i, i}-c_{i+1, j} \widetilde{S}_{i}}{\sqrt{10(j-i) / 3}}+\sqrt{1-\rho} \frac{c_{i+1, j} \widetilde{S}_{i}}{\sqrt{10(j-i) / 3}}+R_{j} \leq y\right) \\
\geq & P\left(Y_{i} \leq x, \sqrt{1-\rho} \frac{S_{j, j}-S_{i, i}-c_{i+1, j} \widetilde{S}_{i}}{\sqrt{10(j-i) / 3}} \leq y\right) \\
& -P\left(y-3 \rho^{1 / 8} \leq \sqrt{1-\rho} \frac{S_{j, j}-S_{i, i}-c_{i+1, j} \widetilde{S}_{i}}{\sqrt{10(j-i) / 3}} \leq y\right)-P\left(\left|\frac{S_{i, i}}{\sqrt{10 j / 3}}\right| \geq \rho^{1 / 8}\right) \\
& -P\left(\left|\sqrt{1-\rho} \frac{c_{i+1, j} \widetilde{S}_{i}}{\sqrt{10(j-i) / 3}}\right| \geq \rho^{1 / 8}\right)-P\left(\left|R_{j}\right| \geq \rho^{1 / 8}\right) \\
\geq & P\left(Y_{i} \leq x, \sqrt{1-\rho} \frac{S_{j, j}-S_{i, i}-c_{i+1, j} \widetilde{S}_{i}}{\sqrt{10(j-i) / 3}} \leq y\right)-\rho^{\tau} \\
= & P\left(Y_{i} \leq x\right) P\left(\sqrt{1-\rho} \frac{S_{j, j}-S_{i, i}-c_{i+1, j} \widetilde{S}_{i}}{\sqrt{10(j-i) / 3}} \leq y\right)-\rho^{\tau} .
\end{aligned}
$$

We can get a similar upper estimate for $P\left(Y_{i} \leq x, Y_{j} \leq y\right)$ in the same way. Thus there exists some constant $M$ such that

$$
P\left(Y_{i} \leq x, Y_{j} \leq y\right)=P\left(Y_{i} \leq x\right) P\left(\sqrt{1-\rho} \frac{S_{j, j}-S_{i, i}-c_{i+1, j} \widetilde{S}_{i}}{\sqrt{10(j-i) / 3}} \leq y\right)+M \rho^{\tau} .
$$

A similar argument,

$$
P\left(Y_{i} \leq x\right) P\left(Y_{j} \leq y\right)=p\left(Y_{i} \leq x\right) P\left(\sqrt{1-\rho} \frac{S_{j, j}-S_{i, i}-c_{i+1, j} \widetilde{S}_{i}}{\sqrt{10(j-i) / 3}} \leq y\right)+M^{\prime} \rho^{\tau},
$$

holds for some constant $M^{\prime}$. Thus we prove that (2.3) holds. 
Let $G_{i, j}(x, y)$ be the joint distribution function of $Y_{i}$ and $Y_{j}$. By (2.2) and (2.3), for $2^{k}<i \leq$ $2^{k+1}, 2^{l}<j \leq 2^{l+1}, l-k \geq 3, l \geq l_{0}$, we can get

$$
\begin{gathered}
\left|\operatorname{Cov}\left(f\left(Y_{i}\right) I\left\{f\left(Y_{i}\right) \leq \frac{k}{(\log k)^{\beta}}\right\}, f\left(Y_{j}\right) I\left\{f\left(Y_{j}\right) \leq \frac{l}{(\log l)^{\beta}}\right\}\right)\right| \\
=\left|\int_{|x| \leq a_{k}} \int_{|y| \leq a_{l}} f(x) f(y) \mathrm{d}\left(G_{i, j}(x, y)-G_{i}(x) G_{j}(y)\right)\right| \\
\ll \frac{k l}{(\log k)^{\beta}(\log l)^{\beta}}\left(\frac{i}{j}\right)^{\tau} \ll \frac{k l}{(\log k)^{\beta}(\log l)^{\beta}} 2^{-(l-k-1) \tau} .
\end{gathered}
$$

Thus we have

$$
\left|\operatorname{Cov}\left(Z_{k}^{*}, Z_{l}^{*}\right)\right| \ll \frac{k l}{(\log k)^{\beta}(\log l)^{\beta}} 2^{-(l-k) \tau} .
$$

We complete the proof of Lemma 2.5 .

Lemma 2.6 Under the conditions of Theorem 1.1, denoting $\eta_{k}=Z_{k}^{*}-\mathbb{E} Z_{k}^{*}$, we have

$$
\mathbb{E}\left(\sum_{k=1}^{N} \eta_{k}\right)^{2}=O\left(\frac{N^{2}}{(\log N)^{2 \beta-1}}\right) .
$$

Proof It follows from Lemma 2.4 and Lemma 2.5 that Lemma 2.6 also holds true. The proof is similar to that of Lemma 4 of Berkes et al. [5]. So we omit it here.

\section{Proof of theorem}

By Lemma 2.6, we have

$$
\mathbb{E}\left(\frac{1}{N} \sum_{k=1}^{N} \eta_{k}\right)^{2}=O\left((\log N)^{1-2 \beta}\right) .
$$

Letting $N_{k}=\left[e^{k \lambda}\right],(2 \beta-1)^{-1}<\lambda<1$, we get

$$
\mathbb{E}\left(\frac{1}{N_{k}} \sum_{k=1}^{N_{k}} \eta_{k}\right)^{2}<\infty,
$$

which implies

$$
\lim _{k \rightarrow \infty} \frac{1}{N_{k}} \sum_{k=1}^{N_{k}} \eta_{k}=0 \quad \text { a.s. }
$$

Note that for $2^{k}<i \leq 2^{k+1}$,

$$
\begin{aligned}
& \mathbb{E} f\left(Y_{i}\right) I\left\{f\left(Y_{i}\right) \leq \frac{k}{(\log k)^{\beta}}\right\} \\
& \quad=\int_{|x| \leq a_{k}} f(x) \mathrm{d} G_{i}(x)=\int_{|x| \leq a_{k}} f(x) \mathrm{d} \Phi\left(\frac{x}{\sigma_{i}}\right)+\int_{|x| \leq a_{k}} f(x) \mathrm{d}\left(G_{i}(x)-\Phi\left(\frac{x}{\sigma_{i}}\right)\right) .
\end{aligned}
$$


Set $a=\int_{-\infty}^{\infty} f(x) \mathrm{d} \Phi(x)$. Noting that $\sigma_{i} \leq 1, \lim _{i \rightarrow \infty} \sigma_{i}=1$, we have

$$
\lim _{k \rightarrow \infty} \sup _{2^{k}<i \leq 2^{k+1}}\left|\int_{|x| \leq a_{k}} f(x) \mathrm{d} \Phi\left(\frac{x}{\sigma_{i}}\right)-a\right|=0
$$

Then by (3.2), (3.3), and (2.2) we get

$$
\begin{aligned}
& \left|\mathbb{E} f\left(Y_{i}\right) I\left\{f\left(Y_{i}\right) \leq \frac{k}{(\log k)^{\beta}}\right\}-a\right| \\
& \quad \leq\left|\int_{|x| \leq a_{k}} f(x) \mathrm{d} \Phi\left(\frac{x}{\sigma_{i}}\right)-a\right|+\left|\int_{|x| \leq a_{k}} f(x) \mathrm{d}\left(G_{i}(x)-\Phi\left(\frac{x}{\sigma_{i}}\right)\right)\right| \\
& \quad \leq o_{k}(1)+\frac{k \theta_{i}}{(\log k)^{\beta}} .
\end{aligned}
$$

Thus

$$
\mathbb{E} Z_{k}^{*}=a \sum_{i=2^{k}+1}^{2^{k+1}} \frac{1}{i}+\zeta_{k} \frac{k}{(\log k)^{\beta}} \sum_{i=2^{k}+1}^{2^{k+1}} \frac{\theta_{i}}{i}+o_{k}(1), \quad\left|\zeta_{k}\right| \leq 1 .
$$

Using $\sum_{i=1}^{L} 1 / i=\log L+O(1)$ and $\sum_{i=1}^{\infty} \frac{\theta_{i}}{i}<\infty$, we get

$$
\begin{aligned}
\left|\frac{\mathbb{E}\left(\sum_{k=1}^{N} Z_{k}^{*}\right)}{\log 2^{N+1}}-a\right| & \ll \frac{1}{N} \sum_{k=1}^{N} \frac{k}{(\log k)^{\beta}} \sum_{i=2^{k}+1}^{2^{k+1}} \frac{\theta_{i}}{i}+o_{N}(1) \\
& =O\left((\log N)^{-\beta}\right)+o_{N}(1) \\
& =o_{N}(1) .
\end{aligned}
$$

Thus by (3.1), we get

$$
\lim _{k \rightarrow \infty} \frac{\sum_{k=1}^{N_{k}} Z_{k}^{*}}{\log 2^{N_{k}+1}}=a \quad \text { a.s. }
$$

Then by Lemma 2.3, we have

$$
\lim _{k \rightarrow \infty} \frac{\sum_{k=1}^{N_{k}} Z_{k}}{\log 2^{N_{k}+1}}=a \quad \text { a.s. }
$$

The relation $\lambda<1$ implies $\lim _{k \rightarrow \infty} N_{k+1} / N_{k}=1$, thus (3.4) and the positivity of the $Z_{k}$ yield

$$
\lim _{N \rightarrow \infty} \frac{\sum_{k=1}^{N} Z_{k}}{\log 2^{N+1}}=a \quad \text { a.s. }
$$

i.e. (1.6) holds for the subsequence $N=2^{k}$. Using again the positivity of the terms, we get (1.6). We complete the proof of Theorem 1.1. 
Authors' contributions

FF conceived of the study and drafted and completed the manuscript. DW participated in the discussion of the manuscript. FF and DW read and approved the final manuscript.

\section{Acknowledgements}

The authors would like to thank the editor and the referees for their very valuable comments by which the quality of the paper has been improved. This research is supported by the National Natural Science Foundation of China (71271042) and the Guangxi China Science Foundation (2013GXNSFAA278003). It is also supported by the Research Project of Guangxi High Institution (YB2014150).

Received: 8 April 2015 Accepted: 1 February 2016 Published online: 09 February 2016

\section{References}

1. Rempala, G, Wesolowski, J: Asymptotics for products of sums and U-statistics. Electron. Commun. Probab. 7, 47-54 (2002)

2. Gonchigdanzan, K, Rempala, G: A note on the almost sure limit theorem for the product of partial sums. Appl. Math. Lett. 19, 191-196 (2006)

3. Tan, ZQ, Peng, ZX: Almost sure central limit theorem for the product of partial sums. Acta Math. Sci. 29, 1689-1698 (2009)

4. Zhang, Y, Yang, XY, Dong, ZS: An almost sure central limit theorem for products of sums of partial sums under association. J. Math. Anal. Appl. 355, 708-716 (2009)

5. Berkes, I, Csáki, E, Horváth, L: Almost sure central limit theorems under minimal conditions. Stat. Probab. Lett. 37, 67-76 (1998)

6. Wu, QY: Almost sure central limit theory for products of sums of partial sums. Appl. Math. J. Chin. Univ. Ser. B 27, 169-180 (2012)

7. Petrov, V: Sums of Independent Random Variables. Springer, New York (1975)

8. Feller, W: The law of iterated logarithm for identically distributed random variables. Ann. Math. 47, 631-638 (1946)

9. Friedman, N, Katz, M, Koopmans, LH: Convergence rates for the central limit theorem. Proc. Natl. Acad. Sci. USA 56, 1062-1065 (1966)

\section{Submit your manuscript to a SpringerOpen ${ }^{\odot}$ journal and benefit from:}

- Convenient online submission

- Rigorous peer review

- Immediate publication on acceptance

Open access: articles freely available online

- High visibility within the field

- Retaining the copyright to your article 